\title{
Are anti-HIV drugs an effective treatment?
}

Although we found Richard Wurtman's medico-political commentary on the development of anti-HIV drugs ${ }^{1}$ informative, we wish to point out that his optimistic scenario for HIV is not real. In fact, it is ironic that a recent US meeting outlining serious limitations of anti-HIV triple therapy, treatment failures and rises in viral levels within four to seven months, was reported under the headline "The Daunting Challenge of Keeping HIV Suppressed" ${ }^{\text {. }}$

Wurtman implies that the drugs are selective for HIV. In fact, they inhibit normal cell division. It is established that AZT causes anemia universally and in the Concorde trial, on extended follow-up its toxicity caused significantly higher mortality. The protease inhibitors are also cytotoxic ${ }^{3}$, even though in some combinations they make a significant improvement in anti-HIV treatment. With regards to elimination of the virus, HIV integrates itself as DNA within the cell's genome where only death of the infected cells can eliminate it; and some lymphocytes have life spans lasting decades.

The repeated suggestion that anti-HIV drugs should be administered as soon after infection as possible, may also be misguided. In most cases the individual immune system manages to contain the damage for many years. Yet all drug treatment is seriously limited by the emergence of resistant mutants and toxicity. So perhaps intervention ought to be initiated only when the virus causes accelerated damage (when the CD4+T-cell count drops below 400). We also suggest that additional treatments should be considered more seriously. For example, there is some evidence to suggest that plasma donation by healthy HIV-infected individuals may delay progress of their disease ${ }^{4}$. The plasma, rich in anti-HIV neutralizing antibodies, provides a non-toxic form of passive immunotherapy for AIDS patients and has been shown to provide statistically significant benefit ${ }^{5}$ with no known adverse effects. Some evidence suggests it may induce clinical remission in patients with AIDS-related complex and arrest the decline in the $\mathrm{CD} 4$ $T$-cell count ${ }^{6}$.

To date, however, the best we can do is prolong the asymptomatic phases of HIV infection by a few years rather that months. This is far from anything that could be called a therapeutic success, let alone justifying Wurtman's claim that "AIDS has been an easy disease to treat."

ABRAHAM Karpas ${ }^{1}$, StePhen ASH $^{2} \&$

DOUGLAS BAINBRIDGE ${ }^{3}$

'Department of Haematology

Cambridge University

$M R C$ Centre Hills Road

Cambridge $\mathrm{CB} 22 \mathrm{QH}$

${ }^{2}$ Ealing Hospital

London UB1 $3 \mathrm{HW}$

${ }^{3}$ Royal London Hospital

Wurtman replies - While the purpose of my article was to analyze the process by which AIDS (as opposed to, for example, Alzheimer's disease or alcoholism or cancer) became treatable, and not to trumpet the success of that treatment, a little trumpeting does seem in order on the basis of recently published data on the extraordinary fall in death rates that can occur when a society makes triple therapy available to all who need it $^{7}$. In British Columbia, where the Centre for Excellence in HIV/AIDS Treatment Programme provides state-of-the-art treatment free of charge, death rates among treated patients declined more than two-thirds, from $18.9 / 1000$ during the first quarter of 1994 to $5.7 / 1000$ during the last quarter of 1996. Among sicker patients with CD4 counts below 100 this decline was comparable (62.0/1000 to $19.8 / 1000)$.

These observations indicate that - contrary to the anxieties expressed by Karpas et al. - triple therapy is effective, and our highest immediate priority should be to guarantee access to it to as many patients as possible. Of course we can assume that our long-term experience with existing anti-HIV drugs will recapitulate that with antibacterials, such as penicillin. Their future use will be limited by the appearance of resistant strains and, in some patients, by unacceptable toxicities. Hence our pharmaceutical industry must be encouraged to continue to provide us with new and better drugs.

\section{RiCHARD J. WURTMAN}

Massachusetts Institute of Technology

Department of Brain and Cognitive Sciences

Room E25-604

Cambridge, Massachusetts 02139-4307, USA

1. Wurtman, R.J. What went right: Why is HIV a treatable infection? Nature Med. 3, 714-717 (1997).

2. Cohen, I. The daunting challenge of keeping HIV suppressed. Science 277, 32-33 (1997).

3. Lipsky, J.J. Antiretroviral drugs for AIDS. Lancet 348, 800-803 (1996).

4. Bainbridge et al. Can repeated plasma donation by asymptomatic HIV-infected individuals delay the onset of AIDS? Phil. Trans. R. Soc. Lond. B. 35, 763-770 (1997).

5. Vittecoq, D. et al. Passive immunotherapy in AIDS: A double-blind randomized study based on transfusions of plasma rich in anti-human immunodeficiency virus 1 antibodies vs. transfusions of seronegative plasma. Proc. Nat. Acad. Sci. USA. 92, 1195-1199 (1995).

6. Karpas, A. et al. Polymerase chain reaction evidence for human immunodeficiency virus-1 neutralized by passive immunotherapy in patients with AIDS and AIDS related complex. Proc. Nat. Acad. Sci. USA. 87, 7613-7617 (1990).

7. Hogg, R.S. et al. Decline in deaths from AIDS due to new antiretrovirals. Lancet 349, 1294 (1997).

\section{CCR2 chemokine receptor and AIDS progression}

Recently we reported an association between the CCR2-64I chemokine receptor mutation and delayed onset of AIDS ${ }^{1}$. Michael et al. have now examined the San Francisco Mens Health Study (SFMHS) AIDS cohort for CCR2 genotypes and in this issue of Nature Medicine report that they fail to detect the CCR2-64I association ${ }^{2}$. Earlier work has shown that in this same SFMHS cohort the previously described association between the CCR5- $\triangle 32$ mutation and de- layed AIDS onset ${ }^{3}$ was found ${ }^{4}$, leading Michael et al. to conclude: "in a cohort adequately powered for the generalizable surrogate markers of viral load and CCR5- $\Delta 32 \ldots$ the association of the CCR2-64I polymorphism may not be generalizable ${ }^{\prime 2}$. We suggest an alternative explanation for their failure to detect the CCR2 association relates to cohort structure and study design.

The SFMHS cohort consists of 495 individuals, enrolled in 1984 , over $90 \%$ of 